\title{
A 2+1-Dimensional Non-Isothermal Magnetogasdynamic System. Hamiltonian-Ermakov Integrable Reduction ${ }^{\star}$
}

\author{
Hongli $A N^{\dagger}$ and Colin ROGERS $\ddagger \S$ \\ † College of Science, Nanjing Agricultural University, Nanjing 210095, P.R. China \\ E-mail:kaixinguoan@163.com \\ $\ddagger$ School of Mathematics and Statistics, The University of New South Wales, \\ Sydney, NSW 2052, Australia \\ $\S$ Australian Research Council Centre of Excellence for Mathematics \& Statistics \\ of Complex Systems, School of Mathematics, The University of New South Wales, \\ Sydney, NSW2052, Australia \\ E-mail: c.rogers@unsw.edu.au \\ URL: http://web. maths.unsw.edu.au/ colinr/
}

Received May 27, 2012, in final form August 02, 2012; Published online August 23, 2012

http://dx.doi.org/10.3842/SIGMA.2012.057

\begin{abstract}
A 2+1-dimensional anisentropic magnetogasdynamic system with a polytropic gas law is shown to admit an integrable elliptic vortex reduction when $\gamma=2$ to a nonlinear dynamical subsystem with underlying integrable Hamiltonian-Ermakov structure. Exact solutions of the magnetogasdynamic system are thereby obtained which describe a rotating elliptic plasma cylinder. The semi-axes of the elliptical cross-section, remarkably, satisfy a Ermakov-Ray-Reid system.
\end{abstract}

Key words: magnetogasdynamic system; elliptic vortex; Hamiltonian-Ermakov structure; Lax pair

2010 Mathematics Subject Classification: 34A34; 35A25

\section{Introduction}

Neukirch et al. $[6,7,8]$ have investigated 2+1-dimensional magnetogasdynamic systems via a solution approach in which the nonlinear acceleration terms in the Lundquist momentum equation either vanish or are conservative. By contrast, in recent work [12, 20] an elliptic vortex ansatz was adopted in 2+1-dimensional isothermal magnetogasdynamics and underlying integrable Ermakov-Ray-Reid structure was isolated. In [20], magnetogasdynamic pulsrodontype solutions were constructed analogous to those originally derived in elliptic warm-core theory in [13]. The pulsrodons describe an elliptical plasma cylinder bounded by a vacuum. The timedependent semi-axes of the elliptical cross-section of the cylinder were shown to be governed by an integrable Hamiltonian Ermakov system.

The present work concerns an extension of that of [12] to a non-isothermal rotating magnetogasdynamic version of a spinning non-conducting gas cloud system with origin in work of Ovsiannikov [9] and Dyson [2]. A nonlinear dynamical subsystem is derived which is again remarkably, shown to have integrable Hamiltonian Ermakov-Ray-Reid structure. Moreover, a Lax pair for the dynamical system is constructed.

\footnotetext{
*This paper is a contribution to the Special Issue "Geometrical Methods in Mathematical Physics". The full collection is available at http://www.emis.de/journals/SIGMA/GMMP2012.html
} 


\section{The magnetogasdynamic system}

Here, we consider a 2+1-dimensional anisentropic magnetogasdynamic system incorporating rotation, namely,

$$
\begin{aligned}
& \frac{\partial \rho}{\partial t}+\operatorname{div}(\rho \mathbf{q})=0 \\
& \rho\left[\frac{\partial \mathbf{q}}{\partial t}+(\mathbf{q} \cdot \nabla) \mathbf{q}+f \mathbf{k} \times \mathbf{q}\right]-\mu \operatorname{curl} \mathbf{H} \times \mathbf{H}+\nabla p=0 \\
& \operatorname{div} \mathbf{H}=0 \\
& \frac{\partial \mathbf{H}}{\partial t}=\operatorname{curl}(\mathbf{q} \times \mathbf{H}) \\
& \frac{\partial S}{\partial t}+\mathbf{q} \cdot \nabla S=0
\end{aligned}
$$

where the velocity $\mathbf{q}$ and magnetic field $\mathbf{H}$ are given by

$$
\mathbf{q}=u \mathbf{i}+v \mathbf{j}, \quad \mathbf{H}=\nabla A \times \mathbf{k}+h \mathbf{k}
$$

respectively, while the gas law adopts the polytropic form

$$
S=-\ln \rho+\frac{1}{\gamma-1} \ln T, \quad \gamma \neq 1
$$

with

$$
p=\rho T \text {. }
$$

In the above, the magneto-gas density $\rho(\mathbf{x}, t)$, pressure $p(\mathbf{x}, t)$, entropy $S(\mathbf{x}, t)$, temperature $T(\mathbf{x}, t)$ and magnetic flux $A(\mathbf{x}, t)$ are all assumed to be dependent only on $\mathbf{x}=x \mathbf{i}+y \mathbf{j}$ and time $t$. In addition, $f$ is the Coriolis constant, $\mu$ the magnetic permeability and $h(\mathbf{x}, t)$ the transverse component of the magnetic field.

Insertion of the representation (2.6) into Faraday's law (2.4) produces the convective constraint

$$
\frac{\partial A}{\partial t}+\mathbf{q} \cdot \nabla A=0
$$

together with

$$
\frac{\partial h}{\partial t}+\operatorname{div}(h \mathbf{q})=0
$$

which holds automatically if we set

$$
h=\lambda \rho, \quad \lambda \in \mathbb{R} .
$$

Here, a novel two-parameter $(m, n)$ pressure-density ansatz

$$
p=\varepsilon_{0}(t) \rho^{2}+\varepsilon_{1}(t) \rho^{n}+\varepsilon_{2}(t) \rho^{m} .
$$

is introduced. In the magnetogasdynamic study of [7], a relation $p \sim \rho$ was adopted, while pressure-density relations of the type $p \sim \rho^{2}$ arise in astrophysical contexts [21]. A parabolic pressure-density law was recently employed in 2+1-dimensional isothermal magnetogasdynamics in [20] and pulsrodon-type solutions were isolated. 
In the present non-isothermal context, substitution of (2.10) in (2.8) produces the temperature distribution

$$
T=\varepsilon_{0}(t) \rho+\varepsilon_{1}(t) \rho^{n-1}+\varepsilon_{2}(t) \rho^{m-1},
$$

while the entropy distribution adopts the form

$$
S=-\ln \rho+\frac{1}{\gamma-1} \ln \left(\varepsilon_{0}(t) \rho+\varepsilon_{1}(t) \rho^{n-1}+\varepsilon_{2}(t) \rho^{m-1}\right) .
$$

The energy equation now requires that

$$
\begin{aligned}
\left(\rho_{t}+\right. & \mathbf{q} \cdot \nabla \rho)\left[\frac{\varepsilon_{0}+(n-1) \varepsilon_{1} \rho^{n-2}+(m-1) \varepsilon_{2} \rho^{m-2}}{(\gamma-1)\left(\varepsilon_{0} \rho+\varepsilon_{1} \rho^{n-1}+\varepsilon_{2} \rho^{m-1}\right)}-\frac{1}{\rho}\right] \\
& +\frac{\dot{\varepsilon}_{0} \rho+\dot{\varepsilon}_{1} \rho^{n-1}+\dot{\varepsilon}_{2} \rho^{m-1}}{(\gamma-1)\left(\varepsilon_{0} \rho+\varepsilon_{1} \rho^{n-1}+\varepsilon_{2} \rho^{m-1}\right)}=0,
\end{aligned}
$$

whence, on use of the continuity equation (2.1)

$$
-\operatorname{div} \mathbf{q}\left[(2-\gamma) \varepsilon_{0} \rho+(n-\gamma) \varepsilon_{1} \rho^{n-1}+(m-\gamma) \varepsilon_{2} \rho^{m-1}\right]+\dot{\varepsilon}_{0} \rho+\dot{\varepsilon}_{1} \rho^{n-1}+\dot{\varepsilon}_{2} \rho^{m-1}=0
$$

that is

$$
\frac{1}{\gamma-1} \frac{\dot{T}}{T}\left[(2-\gamma) \varepsilon_{0} \rho+(n-\gamma) \varepsilon_{1} \rho^{n-1}+(m-\gamma) \varepsilon_{2} \rho^{m-1}\right]+\dot{\varepsilon}_{0} \rho+\dot{\varepsilon}_{1} \rho^{n-1}+\dot{\varepsilon}_{2} \rho^{m-1}=0 .
$$

On substitution of (2.6) and (2.10) into the momentum equation (2.2), it is seen that

$$
\frac{\partial \mathbf{q}}{\partial t}+\mathbf{q} \cdot \nabla \mathbf{q}+f \mathbf{k} \times \mathbf{q}+\frac{1}{\rho}\left[\mu\left(\nabla^{2} A\right)(\nabla A)+\varepsilon_{1} \nabla \rho^{n}\right]+\left(\mu \lambda^{2}+2 \varepsilon_{0}\right) \nabla \rho+\frac{\varepsilon_{2}}{\rho} \nabla \rho^{m}=0
$$

together with

$$
A_{y} \rho_{x}-A_{x} \rho_{y}=0
$$

so that

$$
A=A(\rho, t)
$$

Attention is here restricted to the separable case

$$
A=\Phi(\rho) \Psi(t)
$$

whence, substitution into (2.9) and use of the continuity equation yields

$$
\frac{\dot{\Psi}(t)}{\Psi(t)}=\rho \frac{\Phi^{\prime}(\rho)}{\Phi(\rho)} \operatorname{div} \mathbf{q} .
$$

Here, we proceed with

$$
\Phi=\rho^{n},
$$

where $n$ is the parameter involving in the relation (2.11), so that

$$
\operatorname{div} \mathbf{q}=\left(\frac{1}{n}\right) \frac{\dot{\Psi}}{\Psi}
$$


and

$$
A=\rho^{n} \Psi(t)
$$

Hence, as in the case of the spinning non-conducting gas cloud analysis of Ovsiannikov [9] and Dyson [2], the divergence of the velocity is dependent only on time. Moreover, the relation (2.13) shows that

$$
-\frac{1}{n} \frac{\dot{\Psi}}{\Psi}\left[(2-\gamma) \varepsilon_{0} \rho+(n-\gamma) \varepsilon_{1} \rho^{n-1}+(m-\gamma) \varepsilon_{2} \rho^{m-1}\right]+\dot{\varepsilon}_{0} \rho+\dot{\varepsilon}_{1} \rho^{n-1}+\dot{\varepsilon}_{2} \rho^{m-1}=0
$$

and it is observed that this condition holds identically with

$$
\begin{aligned}
& \varepsilon_{0}=\alpha_{0} \Psi^{\frac{2-\gamma}{n}} \\
& \varepsilon_{1}=\alpha_{1} \Psi^{\frac{n-\gamma}{n}} \\
& \varepsilon_{2}=\alpha_{2} \Psi^{\frac{m-\gamma}{n}}
\end{aligned}
$$

where $\alpha_{i}(i=0,1,2)$ are arbitrary constants of integration. In addition, the isentropic condition (2.5) together with the polytropic gas law (2.7) and the continuity equation (2.1) show that

$$
\operatorname{div} \mathbf{q}=\frac{1}{1-\gamma} \frac{\dot{T}}{T}
$$

whence, on use of (2.11),

$$
(2-\gamma) \operatorname{div} \mathbf{q}=\frac{\dot{\varepsilon}_{0}}{\varepsilon_{0}}, \quad(n-\gamma) \operatorname{div} \mathbf{q}=\frac{\dot{\varepsilon}_{1}}{\varepsilon_{1}}, \quad(m-\gamma) \operatorname{div} \mathbf{q}=\frac{\dot{\varepsilon}_{2}}{\varepsilon_{2}} .
$$

It is seen that in view of (2.14), these relations are indeed consistent with (2.15)-(2.17).

In summary, the magnetogasdynamic system now reduces to consideration of the nonlinear coupled system

$$
\begin{aligned}
& \frac{\partial \rho}{\partial t}+\operatorname{div}(\rho \mathbf{q})=0, \\
& \frac{\dot{\Psi}}{\Psi}=n \operatorname{div} \mathbf{q}, \\
& \frac{\partial \mathbf{q}}{\partial t}+\mathbf{q} \cdot \nabla \mathbf{q}+f \mathbf{k} \times \mathbf{q}+\frac{1}{\rho}\left(\mu \Psi^{2} \nabla^{2} \rho^{n}+\varepsilon_{1}\right) \nabla \rho^{n}+\left(\mu \lambda^{2}+2 \varepsilon_{0}\right) \nabla \rho+\frac{\varepsilon_{2} m}{m-1} \nabla \rho^{m-1}=0,
\end{aligned}
$$

where $m \neq 1$, together with the additional conditions (2.15)-(2.17). The inherent nonlinearity of the system (2.18) remains a major impediment to analytic progress. It is noted also that the system $(2.18)_{1,3}$ is overdetermined since it is implicitly constrained by the requirement $(2.18)_{2}$ that div $\mathbf{q}$ be a function of $t$ only.

\section{An elliptic vortex ansatz. A dynamical system reduction}

Here, integrable nonlinear dynamical subsystems of the magnetogasdynamic system (2.18) are sought via an elliptic vortex ansatz of the type

$$
\mathbf{q}=\mathbf{L}(t) \mathbf{x}+\mathbf{M}(t), \quad \rho=\left(\mathbf{x}^{T} \mathbf{E}(t) \mathbf{x}+\rho_{0}\right)^{m-1}, \quad m \neq 1, \quad \mathbf{x}=\left(\begin{array}{l}
x-\bar{q}(t) \\
y-\bar{p}(t)
\end{array}\right)
$$


with

$$
\mathbf{L}(t)=\left(\begin{array}{ll}
u_{1}(t) & u_{2}(t) \\
v_{1}(t) & v_{2}(t)
\end{array}\right), \quad \mathbf{E}(t)=\left(\begin{array}{cc}
a(t) & b(t) \\
b(t) & c(t)
\end{array}\right), \quad \mathbf{M}(t)=\left(\begin{array}{c}
\dot{\bar{q}}(t) \\
\dot{\bar{p}}(t)
\end{array}\right)
$$

Insertion of (3.1) into the continuity equation yields

$$
\left(\begin{array}{c}
\dot{a} \\
\dot{b} \\
\dot{c}
\end{array}\right)+\left(\begin{array}{ccc}
2 u_{1}+(m-1)\left(u_{1}+v_{2}\right) & 2 v_{1} & 0 \\
u_{2} & m\left(u_{1}+v_{2}\right) & v_{1} \\
0 & 2 u_{2} & 2 v_{2}+(m-1)\left(u_{1}+v_{2}\right)
\end{array}\right)\left(\begin{array}{l}
a \\
b \\
c
\end{array}\right)=\mathbf{0}
$$

together with

$$
\dot{\rho}_{0}+\rho_{0}(m-1)\left(u_{1}+v_{2}\right)=0,
$$

whence

$$
\rho_{0}=\operatorname{const} \Psi^{(1-m) / n} .
$$

If we now proceed with

$$
n=m-1
$$

together with

$$
2 \varepsilon_{0}+\mu \lambda^{2}=0
$$

and

$$
\varepsilon_{1}+2 \mu \Psi^{2}(a+c)=0
$$

then it is seen that $(2.18)_{3}$ reduces to

$$
\frac{\partial \mathbf{q}}{\partial t}+\mathbf{q} \cdot \nabla \mathbf{q}+f \mathbf{k} \times \mathbf{q}+\frac{m}{m-1} \varepsilon_{2} \nabla \rho^{m-1}=0 .
$$

The relation (3.7) implies that $\dot{\varepsilon}_{0}=0$ whence (2.15) shows that the adiabatic index $\gamma=2$, while (3.8) and (2.16) together require

$$
a+c=-\frac{\alpha_{1}}{2 \mu} \Psi^{-(n+2) / n}=-\frac{\alpha_{1}}{2 \mu} \Psi^{(1+m) /(1-m)}, \quad n \neq 1 .
$$

Substitution of (3.1) into (3.9) now gives

$$
\left(\begin{array}{c}
\dot{u}_{1} \\
\dot{u}_{2} \\
\dot{v}_{1} \\
\dot{v}_{2}
\end{array}\right)+\left(\begin{array}{cc}
\mathbf{L}^{T} & -f \mathbf{I} \\
f \mathbf{I} & \mathbf{L}^{T}
\end{array}\right)\left(\begin{array}{l}
u_{1} \\
u_{2} \\
v_{1} \\
v_{2}
\end{array}\right)+2 \varepsilon_{2} \frac{m}{m-1}\left(\begin{array}{l}
a \\
b \\
b \\
c
\end{array}\right)=\mathbf{0}
$$

augmented by the auxiliary linear equations

$$
\ddot{\bar{p}}+f \dot{\bar{q}}=0, \quad \ddot{\bar{q}}-f \dot{\bar{p}}=0 .
$$

It is noted that the relation $(2.18)_{2}$ together with (3.6) shows that

$$
\dot{\Psi}=(m-1)\left(u_{1}+v_{2}\right) \Psi .
$$


While $\rho_{0}$ is given in terms of $\Psi$ via (3.5). The constraints (3.7) and (3.8) are to be adjoined and their admissibility will be examined subsequently.

In what follows, it proves convenient to proceed in terms of new variables, namely

$$
\begin{aligned}
& G=u_{1}+v_{2}, \quad G_{R}=\frac{1}{2}\left(v_{1}-u_{2}\right), \quad G_{S}=\frac{1}{2}\left(v_{1}+u_{2}\right), \quad G_{N}=\frac{1}{2}\left(u_{1}-v_{2}\right), \\
& B=a+c, \quad B_{S}=b, \quad B_{N}=\frac{1}{2}(a-c) .
\end{aligned}
$$

These quantities were originally introduced in a hydrodynamic context (see e.g. [13]). Therein, $G$ and $G_{R}$ correspond, in turn, to the divergence and spin of the velocity field, while $G_{S}$ and $G_{N}$ represent shear and normal deformation rates. The system (3.3) and (3.4) together with (3.10) now reduces to the nonlinear dynamical system

$$
\begin{aligned}
& \dot{\rho}_{0}+(m-1) \rho_{0} G=0, \\
& \dot{B}+m B G+4\left(B_{N} G_{N}+B_{S} G_{S}\right)=0, \\
& \dot{B}_{S}+m B_{S} G+B G_{S}-2 B_{N} G_{R}=0, \\
& \dot{B}_{N}+m B_{N} G+B G_{N}+2 B_{S} G_{R}=0, \\
& \dot{G}+\frac{1}{2} G^{2}+2\left(G_{N}^{2}+G_{S}^{2}-G_{R}^{2}\right)-2 f G_{R}+2 \frac{\varepsilon_{2} m}{m-1} B=0, \\
& \dot{G}_{N}+G G_{N}-f G_{S}+2 \frac{\varepsilon_{2} m}{m-1} B_{N}=0, \\
& \dot{G}_{S}+G G_{S}+f G_{N}+2 \frac{\varepsilon_{2} m}{m-1} B_{S}=0, \\
& \dot{G}_{R}+G G_{R}+\frac{1}{2} f G=0
\end{aligned}
$$

together with

$$
\dot{\Psi}=(m-1) \Psi G .
$$

It is observed that the introduction of the pressure-density parameters $(m, n)$ and $\varepsilon_{2}$ leads to a generalisation of the nonlinear dynamical systems obtained in $[12,13,14,20]$.

If we now introduce the quantity $\Omega$ via

$$
G=\frac{2 \dot{\Omega}}{\Omega}
$$

then $(3.12)_{1}$ and $(3.12)_{8}$ show, in turn, that

$$
\rho_{0}=\frac{c_{\mathrm{I}}}{\Omega^{2(m-1)}}
$$

and

$$
G_{R}=\frac{c_{0}}{\Omega^{2}}-\frac{1}{2} f
$$

While the relation (3.13) yields

$$
\Psi=\nu \Omega^{2(m-1)},
$$

where $c_{0}, c_{\mathrm{I}}$ and $\nu$ denote arbitrary constants of integration.

Two conditions which are key to the subsequent development and which may be established by appeal to the original system (3.12) are now recorded. These represent extensions of results obtained in a hydrodynamic context $[13,14]$. 


\section{Theorem 1.}

$$
\dot{M}+(m+1) G M=0, \quad \dot{Q}+(m+1) G Q=0,
$$

where

$$
\begin{aligned}
M= & 2\left(B_{N} G_{S}-B_{S} G_{N}\right)-B\left(G_{R}+\frac{1}{2} f\right), \quad \triangle=\frac{1}{4} B^{2}-B_{S}^{2}-B_{N}^{2}, \\
Q= & -B\left(G_{S}^{2}+G_{N}^{2}+G_{R}^{2}+\frac{1}{4} G^{2}\right)+4 G_{R}\left(B_{N} G_{S}-B_{S} G_{N}\right) \\
& +2 G\left(B_{S} G_{S}+B_{N} G_{N}\right)+4 \frac{\varepsilon_{2} m}{m-1} \triangle-4 \frac{m}{m-1} \triangle \Omega^{m-1} \int \dot{\varepsilon}_{2} \Omega^{1-m} d t .
\end{aligned}
$$

New $\Omega$-modulated variables involving the pressure-density parameter $m$ are now introduced according to

$$
\bar{B}=\Omega^{2 m} B, \quad \bar{B}_{S}=\Omega^{2 m} B_{S}, \quad \bar{B}_{N}=\Omega^{2 m} B_{N}, \quad \bar{G}_{S}=\Omega^{2} G_{S}, \quad \bar{G}_{N}=\Omega^{2} G_{N},
$$

whence the dynamical system (3.12) reduces to

$$
\begin{aligned}
& \dot{\bar{B}}+\frac{4\left(\bar{B}_{N} \bar{G}_{N}+\bar{B}_{S} \bar{G}_{S}\right)}{\Omega^{2}}=0, \\
& \dot{\bar{B}}_{S}+f \bar{B}_{N}+\frac{\bar{B} \bar{G}_{S}-2 c_{0} \bar{B}_{N}}{\Omega^{2}}=0, \\
& \dot{\bar{B}}_{N}-f \bar{B}_{S}+\frac{\bar{B} \bar{G}_{N}+2 c_{0} \bar{B}_{S}}{\Omega^{2}}=0, \\
& \dot{\bar{G}}_{S}+f \bar{G}_{N}+\frac{2 \varepsilon_{2} m}{m-1} \frac{\bar{B}_{S}}{\Omega^{2(m-1)}}=0, \\
& \dot{\bar{G}}_{N}-f \bar{G}_{S}+\frac{2 \varepsilon_{2} m}{m-1} \frac{\bar{B}_{N}}{\Omega^{2(m-1)}}=0
\end{aligned}
$$

augmented by the relations (3.14) and (3.15) together with a nonlinear equation for $\Omega$, namely

$$
\Omega^{3} \ddot{\Omega}+\frac{1}{4} f^{2} \Omega^{4}+\bar{G}_{N}^{2}+\bar{G}_{S}^{2}-c_{0}^{2}+\frac{\varepsilon_{2} m}{m-1} \frac{\bar{B}}{\Omega^{2(m-2)}}=0 .
$$

The reduced dynamical system (3.18) together with (3.19) and the constraints given by (2.15)(2.17) and (3.7), (3.8) will now be examined in detail.

Thus, if we turn to the expressions for $\varepsilon_{0}, \varepsilon_{1}$ and $\varepsilon_{2}$ as given by (2.15)-(2.17), it is seen immediately that consistency of (2.15) and (3.7) requires that the adiabatic index $\gamma=2$. Further, comparison of the expressions for $\varepsilon_{1}$ in (2.16) and (3.8) now yields

$$
\alpha_{1} \Psi^{\frac{n-2}{n}}+2 \mu \Psi^{2}(a+c)=0,
$$

where the relations (3.6), (3.16) and (3.17) combine to show that

$$
\alpha_{1} \nu^{\frac{m-3}{m-1}}+2 \mu \nu^{2} \Omega^{2} \bar{B}=0
$$

whence

$$
\nu=0 \quad \text { or } \quad \Omega^{2} \bar{B}=-\frac{\alpha_{1}}{2 \mu} \nu^{\frac{1+m}{1-m}}:=\delta .
$$

In the former case, by virtue of (3.16), the magnetic flux $A$ vanishes so that the magnetic field $\mathbf{H}$ is purely transverse and the dynamical system (3.18) and (3.19) is not thereby constrained. Here, 
we proceed with the latter case, so that the system (3.18) and (3.19) is additionally constrained by the requirement $\Omega^{2} \bar{B}=$ const and $(3.18)_{1}$ yields

$$
\bar{B}_{N} \bar{G}_{N}+\bar{B}_{S} \bar{G}_{S}-\frac{\delta \dot{\Omega}}{2 \Omega}=0
$$

Finally, for $\varepsilon_{2}$, the relations (2.17), (3.6) and (3.16) combine to show that

$$
\varepsilon_{2}=\alpha_{2} \nu^{\frac{m-2}{m-1}} \Omega^{2(m-2)}
$$

which it subsequently proves convenient to re-write in this form

$$
\varepsilon_{2}=\alpha\left(\frac{m-1}{m}\right) \Omega^{2(m-2)} .
$$

\section{Integrals of motion and parametrisation}

Under the constraint (3.20), the nonlinear dynamical system (3.18) is readily shown to admit the key integrals of motion

$$
\begin{aligned}
& \bar{B}_{S}^{2}+\bar{B}_{N}^{2}-\frac{1}{4} \bar{B}^{2}=c_{\mathrm{II}}, \\
& \bar{G}_{S}^{2}+\bar{G}_{N}^{2}-\alpha \bar{B}=c_{\mathrm{III}}, \\
& 2\left(\bar{B}_{N} \bar{G}_{S}-\bar{B}_{S} \bar{G}_{N}\right)-c_{0} \bar{B}=c_{\mathrm{IV}}, \\
& 2\left(G_{R}+c_{0} \bar{B}\right)+2 G\left(\bar{B}_{S} \bar{G}_{S}+\bar{B}_{N} \bar{G}_{N}\right)+4 \alpha c_{\mathrm{II}} \Omega^{-2} \frac{m-1}{m-3} \\
& \quad-\bar{B} \Omega^{2}\left(\frac{\bar{G}_{S}^{2}+\bar{G}_{N}^{2}}{\Omega^{4}}+\frac{G^{2}}{4}+G_{R}^{2}\right)=c_{\mathrm{V}},
\end{aligned}
$$

where $c_{\mathrm{II}}, c_{\mathrm{III}}, c_{\mathrm{IV}}$ and $c_{\mathrm{V}}$ are constants of integration.

The relations (4.1) and (4.2) may be conveniently parametrised according to

$$
\begin{array}{ll}
\bar{B}_{S}=-\sqrt{c_{\mathrm{II}}+\frac{1}{4} \bar{B}^{2}} \cos \phi(t), & \bar{B}_{N}=-\sqrt{c_{\mathrm{II}}+\frac{1}{4} \bar{B}^{2}} \sin \phi(t), \\
\bar{G}_{S}=-\sqrt{c_{\mathrm{III}}+\bar{B}} \sin \theta(t), & \bar{G}_{N}=+\sqrt{c_{\mathrm{III}}+\bar{B}} \cos \theta(t) .
\end{array}
$$

Substitution of this parametrisation into $(3.18)_{1}$ yields

$$
\dot{\bar{B}}+\frac{4}{\Omega^{2}} \sqrt{\left(c_{\mathrm{II}}+\bar{B}^{2} / 4\right)\left(c_{\mathrm{III}}+\alpha \bar{B}\right)} \sin (\theta-\phi)=0,
$$

while conditions $(3.18)_{2,3}$ reduce to a single relation, namely

$$
\left(\dot{\phi}-f+\frac{2 c_{0}}{\Omega^{2}}\right) \sqrt{c_{\mathrm{II}}+\frac{1}{4} \bar{B}^{2}}-\frac{\bar{B}}{\Omega^{2}} \sqrt{c_{\mathrm{III}}+\alpha \bar{B}} \cos (\theta-\phi)=0 .
$$

Similarly, (3.18) 4,5 produce another single requirement

$$
(\dot{\theta}-f) \sqrt{c_{\mathrm{III}}+\alpha \bar{B}}+\frac{2 \alpha}{\Omega^{2}} \sqrt{c_{\mathrm{II}}+\frac{1}{4} \bar{B}^{2}} \cos (\theta-\phi)=0 .
$$

Moreover, substitution of the representations (4.5) into (4.3) yields

$$
c_{0} \bar{B}=-c_{\mathrm{IV}}+2 \sqrt{\left(c_{\mathrm{II}}+\bar{B}^{2} / 4\right)\left(c_{\mathrm{III}}+\alpha \bar{B}\right)} \cos (\theta-\phi),
$$


while elimination of $\theta-\phi$ in (4.7) and (4.8) respectively shows that

$$
\dot{\phi}=f+\frac{2}{\Omega^{2}}\left[\frac{\delta\left(c_{0} \delta+c_{\mathrm{IV}} \Omega^{2}\right)}{\delta^{2}+4 c_{\mathrm{II}} \Omega^{4}}-c_{0}\right]
$$

and

$$
\dot{\theta}=f-\frac{\alpha}{\Omega^{2}}\left[\frac{c_{0} \delta+c_{\mathrm{IV}} \Omega^{2}}{\alpha \delta^{2}+c_{\mathrm{III}} \Omega^{2}}\right] .
$$

It remains to consider the nonlinear equation (3.19) for $\Omega$, namely

$$
\Omega^{3} \ddot{\Omega}+\frac{f^{2}}{4} \Omega^{4}+c_{\mathrm{III}}-c_{0}^{2}+\frac{\varepsilon_{2} m}{m-1} \frac{\bar{B}}{\Omega^{2(m-2)}}=0
$$

which, by virtue of (3.20), reduces to a generalisation of the classical Steen-Ermakov equation [3, 22], namely

$$
\ddot{\Omega}+\frac{1}{4} f^{2} \Omega=\frac{c_{0}^{2}-c_{\mathrm{III}}}{\Omega^{3}}-\frac{2 \alpha \delta}{\Omega^{5}} .
$$

Further, on use of Theorem 1, it may be readily shown that there is the necessary requirement (cf. [13])

$$
\left(\Omega^{2} \bar{B}\right)+f^{2} \Omega^{2} \bar{B}=-2\left(Q^{*}+f M^{*}\right) \Omega^{2(m+1)}=-2\left(c_{\mathrm{V}}+f c_{\mathrm{IV}}\right) .
$$

This holds automatically here with

$$
\Omega^{2} \bar{B}=\text { const }=\delta=-2\left(c_{\mathrm{V}}+f c_{\mathrm{IV}}\right) / f^{2}, \quad f \neq 0 .
$$

Elimination of $\theta-\phi$ between (4.6) and (4.9) now yields

$$
\dot{\bar{B}}^{2}+\frac{4}{\Omega^{4}}\left(c_{0} \bar{B}+c_{\mathrm{IV}}\right)^{2}=\frac{4}{\Omega^{4}}\left(4 c_{\mathrm{II}}+\bar{B}^{2}\right)\left(c_{\mathrm{III}}+\alpha \bar{B}\right),
$$

whence on use of (4.13)

$$
\delta^{2} \dot{\Omega}^{2}+\frac{\left(c_{0}^{2}-c_{\mathrm{III}}\right) \delta^{2}}{\Omega^{2}}+\left(c_{\mathrm{IV}}^{2}-4 c_{\mathrm{II}} c_{\mathrm{III}}\right) \Omega^{2}-\frac{\alpha \delta^{3}}{\Omega^{4}}+2 c_{0} c_{\mathrm{IV}}-4 \alpha c_{\mathrm{II}} \delta=0 .
$$

The latter equation is required to be compatible with the $1^{\text {st }}$ integral of (4.12), namely

$$
\dot{\Omega}^{2}+\frac{1}{4} f^{2} \Omega^{2}+\frac{\left(c_{0}^{2}-c_{\mathrm{III}}\right)}{\Omega^{2}}-\frac{\alpha \delta}{\Omega^{4}}+k=0
$$

and these are indeed seen to be consistent subject to the relations

$$
c_{\mathrm{IV}}^{2}-4 c_{\mathrm{II}} c_{\mathrm{III}}=\frac{\delta^{2} f^{2}}{4}
$$

and

$$
k=\frac{2 c_{0} c_{\mathrm{IV}}-4 \alpha c_{\mathrm{II}} \delta}{\delta^{2}} .
$$

In summary, a multi-parameter class of exact vortex solutions of the original 2+1-dimensional magnetogasdynamic system has been generated with the velocity components $u_{1}, u_{2}, v_{1}, v_{2}$ and the quantities $a, b, c, \rho_{0}$ in the density representation given, in turn, by

$$
\begin{aligned}
& u_{1}=\frac{\dot{\Omega}}{\Omega}+\frac{1}{\Omega^{3}} \sqrt{\alpha \delta+c_{\mathrm{III}} \Omega^{2}} \cos \theta(t), \quad v_{1}=\frac{c_{0}}{\Omega^{2}}-\frac{f}{2}-\frac{1}{\Omega^{3}} \sqrt{\alpha \delta+c_{\mathrm{III}} \Omega^{2}} \sin \theta(t), \\
& u_{2}=-\frac{c_{0}}{\Omega^{2}}+\frac{f}{2}-\frac{1}{\Omega^{3}} \sqrt{\alpha \delta+c_{\mathrm{III}} \Omega^{2}} \sin \theta(t), \quad v_{2}=\frac{\dot{\Omega}}{\Omega}-\frac{1}{\Omega^{2}} \sqrt{\alpha \delta+c_{\mathrm{III}} \Omega^{2}} \cos \theta(t)
\end{aligned}
$$


together with

$$
\begin{array}{lll}
a=\frac{1}{2 \Omega^{2(m+1)}}\left[\delta-\sqrt{4 c_{\mathrm{II}} \Omega^{4}+\delta^{2}} \sin \phi(t)\right], & b=\frac{1}{2 \Omega^{2(m+1)}} \sqrt{4 c_{\mathrm{II}} \Omega^{4}+\delta^{2}} \cos \phi(t), \\
c=\frac{1}{\Omega^{2(m+1)}}\left[\delta+\sqrt{4 c_{\mathrm{II}} \Omega^{4}+\delta^{2}} \sin \phi(t)\right], & \rho_{0}=\frac{c_{\mathrm{I}}}{\Omega^{2(m-1)}},
\end{array}
$$

where the angles $\phi$ and $\theta$ are obtained by integration of (4.10) and (4.11), respectively while $\Omega$ is given by an elliptic integral resulting from (4.14).

The magnetic flux $A$ is given by

$$
A=\nu \rho^{m-1} \Omega^{2(m-1)}=\nu\left[a(x-\bar{q})^{2}+2 b(x-\bar{q})(y-\bar{p})+c(y-\bar{p})^{2}+\rho_{0}\right] \Omega^{2(m-1)},
$$

while the temperature $T$ and entropy distribution $S$ are determined by (2.11) and (2.12), respectively.

\section{$5 \quad$ Hamiltonian Ermakov structure}

The nonlinear dynamical system (3.12) may be shown to have remarkable underlying structure in that it will be seen to reduce to consideration of an integrable Ermakov-Ray-Reid type system

$$
\ddot{\alpha}+\omega^{2}(t) \alpha=\frac{1}{\alpha^{2} \beta} F(\beta / \alpha), \quad \ddot{\beta}+\omega^{2}(t) \beta=\frac{1}{\alpha \beta^{2}} G(\alpha / \beta) .
$$

Such systems have their origin in the work of Ermakov [3] and were introduced by Ray and Reid in $[10,11]$. Extension to 2+1-dimensions were presented in [15] and to multi-component systems in [18]. The main theoretical interest in the system resides in its admittance of a distinctive integral of motion, namely, the Ray-Reid invariant

$$
I=\frac{1}{2}(\alpha \dot{\beta}-\beta \dot{\alpha})^{2}+\int^{\beta / \alpha} F(z) d z+\int^{\alpha / \beta} G(w) d w .
$$

Ermakov-Ray-Reid systems arise, in particular, in a variety of contexts in nonlinear optics (see e.g. $[16,17]$ and references cited therein).

Here, we proceed with $\bar{p}(t)=\bar{q}(t)=0$ in the ansatz (3.1), since the translation terms $\bar{p}(t), \bar{q}(t)$ are readily re-introduced by use of a Lie group invariance of the magnetogasdynamic system.

The semi-axes of the time-modulated ellipse

$$
a(t) x^{2}+2 b(t) x y+c y^{2}+h_{0}(t)=0, \quad a c-b^{2}>0,
$$

are now given by

$$
\Phi=\sqrt{\frac{2 \rho_{0}}{\sqrt{(a-c)^{2}+4 b^{2}}-(a+c)}}=\sqrt{-\frac{c_{\mathrm{I}}}{2 c_{\mathrm{II}}}} \sqrt{-\delta-\sqrt{4 c_{\mathrm{II}} \Omega^{4}+\delta^{2}}}
$$

and

$$
\Psi=\sqrt{\frac{2 \rho_{0}}{-\sqrt{(a-c)^{2}+4 b^{2}}-(a+c)}}=\sqrt{-\frac{c_{\mathrm{I}}}{2 c_{\mathrm{II}}}} \sqrt{-\delta+\sqrt{4 c_{\mathrm{II}} \Omega^{4}+\delta^{2}}},
$$

where it is required that

$$
c_{\mathrm{I}}>0, \quad c_{\mathrm{II}}<0, \quad \delta<0, \quad \delta^{2}+4 c_{\mathrm{II}} \Omega^{4}>0 .
$$


It is readily established that the semi-axes $\Phi, \Psi$ are governed by a Ermakov-Ray-Reid system, namely

$$
\begin{aligned}
& \ddot{\Phi}+\frac{1}{4} f^{2} \Phi=\frac{1}{\Phi^{2} \Psi}\left[\frac{Z Z^{\prime}}{1+(\Psi / \Phi)^{2}}-\left(\frac{\Psi}{\Phi}\right) \frac{\left(Z^{2}+k / 4\right)}{\left[1+(\Psi / \Phi)^{2}\right]^{2}}\right], \\
& \ddot{\Psi}+\frac{1}{4} f^{2} \Psi=\frac{1}{\Phi \Psi^{2}}\left[-\frac{Z Z^{\prime}}{1+(\Psi / \Phi)^{2}}-\left(\frac{\Phi}{\Psi}\right) \frac{\left(Z^{2}+k / 4\right)}{\left[1+(\Phi / \Psi)^{2}\right]^{2}}\right],
\end{aligned}
$$

where

$$
Z=Z(\Phi / \Psi)=\Psi \dot{\Phi}-\dot{\Psi} \Phi=\frac{2 c_{\mathrm{I}}}{\Omega \sqrt{-c_{\mathrm{II}}}} \sqrt{\frac{\left(\delta^{2}+4 c_{\mathrm{II}} \Omega^{4}\right)\left(\alpha \delta+c_{\mathrm{III}} \Omega^{2}\right)-\Omega^{2}\left(c_{0} \delta+c_{\mathrm{IV}} \Omega^{2}\right)^{2}}{\delta^{2}+4 c_{\mathrm{II}} \Omega^{4}}}
$$

and $\Omega$ is given in terms of the ratio of the semi-axes via the relation

$$
\Omega=\left(-\frac{\delta^{2}}{c_{\mathrm{II}}}\right)^{1 / 4}\left(\frac{\Psi}{\Phi}+\frac{\Phi}{\Psi}\right)^{-1 / 2}
$$

In addition, the Ermakov-Ray-Reid system (5.1) is seen to be Hamiltonian with invariant

$$
H=\frac{1}{2}\left(\dot{\Phi}^{2}+\dot{\Psi}^{2}\right)-\frac{1}{2\left(\Phi^{2}+\Psi^{2}\right)}\left[Z^{2}-\frac{f^{2}}{4}\left(\Phi^{2}+\Psi^{2}\right)^{2}+\frac{k}{4}\right]=-\frac{1}{4} f^{2} \frac{c_{\mathrm{I}} c_{\mathrm{Iv}}}{c_{\mathrm{II}}}, \quad c_{\mathrm{II}} \neq 0,
$$

and accordingly, is amenable to the general procedure described in detail in [14].

It is remarkable indeed that the semi-axes $\Phi$ and $\Psi$ of the time modulated ellipse associated with the density representation in (3.1), are governed by an integrable Ermakov-Ray-Reid system, albeit of some complexity. In fact, a Ermakov-Ray-Reid system may also be associated with the velocity components, at least, in a particular reduction. Attention is here restricted, as in the work of Dyson [2] on non-conducting gas clouds, to irrotational motions in the absence of a Coriolis term.

Thus, here we set

$$
\mathbf{L}=\left(\begin{array}{cc}
\dot{\alpha}(t) / \alpha(t) & 0 \\
0 & \dot{\beta}(t) / \beta(t)
\end{array}\right), \quad \mathbf{E}=\left(\begin{array}{cc}
a(t) & 0 \\
0 & c(t)
\end{array}\right)
$$

in (3.2) corresponding to the subclass of exact solutions in (4.15) with $\theta=0, \phi=\pi / 2$ and

$$
\begin{aligned}
& \frac{\dot{\alpha}}{\alpha}=\frac{\dot{\Omega}}{\Omega}+\frac{1}{\Omega^{2}} \sqrt{c_{\mathrm{III}}+\frac{\alpha \delta}{\Omega^{2}}}, \quad \frac{\dot{\beta}}{\beta}=\frac{\dot{\Omega}}{\Omega}-\frac{1}{\Omega^{2}} \sqrt{c_{\mathrm{III}}+\frac{\alpha \delta}{\Omega^{2}}}, \\
& a=\frac{1}{2 \Omega^{2(m+1)}}\left[\delta-\sqrt{4 c_{\mathrm{II}} \Omega^{4}+\delta^{2}}\right], \quad c=\frac{1}{2 \Omega^{2(m+1)}}\left[\delta+\sqrt{4 c_{\mathrm{II}} \Omega^{4}+\delta^{2}}\right] .
\end{aligned}
$$

The continuity equation, via (3.3), yields

$$
\frac{\dot{a}}{a}+\frac{\dot{\alpha}}{\alpha}(m+1)+\frac{\dot{\beta}}{\beta}(m-1)=0, \quad \frac{\dot{c}}{c}+\frac{\dot{\alpha}}{\alpha}(m-1)+\frac{\dot{\beta}}{\beta}(m+1)=0,
$$

whence

$$
a=c_{\mathrm{I}} \alpha^{-(m+1)} \beta^{1-m}, \quad c=c_{\mathrm{II}} \alpha^{1-m} \beta^{-(m+1)} .
$$

Moreover, (3.4) shows that

$$
\rho_{0}=c_{\mathrm{III}}(\alpha \beta)^{1-m}=c_{\mathrm{III}}^{*} \Omega^{2(1-m)} .
$$


In the above, $c_{\mathrm{I}}, c_{\mathrm{II}}, c_{\mathrm{III}}$ and $c_{\mathrm{III}}^{*}$ are arbitrary non-zero constants of integration. The momentum equation gives

$$
\ddot{\alpha}+2 \varepsilon_{2}(t) \frac{m}{m-1} a \alpha=0, \quad \ddot{\beta}+2 \varepsilon_{2}(t) \frac{m}{m-1} c \beta=0
$$

together with

$$
\ddot{\bar{p}}=0, \quad \ddot{\bar{q}}=0 .
$$

Insertion of the expressions (5.2) into (5.3) gives

$$
\ddot{\alpha}+2 \varepsilon_{2}(t) \frac{m}{m-1} \frac{c_{\mathrm{I}}}{\alpha^{2} \beta}(\alpha \beta)^{2-m}=0, \quad \ddot{\beta}+2 \varepsilon_{2}(t) \frac{m}{m-1} \frac{c_{\mathrm{II}}}{\alpha \beta^{2}}(\alpha \beta)^{2-m}=0,
$$

whence, in view of the relation (3.20), we again obtain a Ermakov-Ray-Reid system, namely

$$
\ddot{\alpha}=\frac{c_{\mathrm{I}}^{*}}{\alpha^{2} \beta}, \quad \ddot{\beta}=\frac{c_{\mathrm{II}}^{*}}{\alpha \beta^{2}},
$$

with the Ray-Reid invariant

$$
I=\frac{1}{2}(\dot{\alpha} \beta-\alpha \dot{\beta})^{2}+c_{\mathrm{I}}^{*} \frac{\beta}{\alpha}+c_{\mathrm{II}}^{*} \frac{\alpha}{\beta},
$$

where

$$
c_{\mathrm{I}}^{*}=-2 \alpha c_{\mathrm{I}}\left(\frac{c_{\mathrm{III}}^{*}}{c_{\mathrm{III}}}\right)^{\frac{m-2}{m-1}}, \quad c_{\mathrm{II}}^{*}=-2 \alpha c_{\mathrm{II}}\left(\frac{c_{\mathrm{III}}^{*}}{c_{\mathrm{III}}}\right)^{\frac{m-2}{m-1}} .
$$

It is observed moreover, that the system (5.4) is also Hamiltonian with additional integral of motion

$$
H=\frac{1}{2}\left(c_{\mathrm{I}} \dot{\beta}^{2}+c_{\mathrm{II}} \dot{\alpha}^{2}\right)+\frac{c_{\mathrm{I}}^{*} c_{\mathrm{II}}^{*}}{\alpha \beta} .
$$

\section{A Lax pair formulation}

It is now shown, following a procedure analogous to that set down in the spinning gas cloud analysis of [16], that the nonlinear dynamical system (3.3) and (3.10) admits an associated Lax pair representation. In this connection, it is seen that the nonlinear dynamical system given by (3.3) together with (3.10) arising from the ansatz (3.1) and (3.2) may be written in the compact matrix form as

$$
\begin{aligned}
& \dot{\mathbf{E}}+\mathbf{E} \mathbf{L}+\mathbf{L}^{T} \mathbf{E}+(m-1) \mathbf{E} \operatorname{tr} \mathbf{L}=\mathbf{0}, \\
& \dot{\mathbf{L}}+\mathbf{L}^{2}+f \mathbf{P L}+2 \varepsilon_{2}(t) \frac{m}{m-1} \mathbf{E}=\mathbf{0}, \quad m \neq 1,
\end{aligned}
$$

where $\mathbf{L}, \mathbf{E}$ are given by (3.2) and

$$
\mathbf{P}=\left(\begin{array}{cc}
0 & -1 \\
1 & 0
\end{array}\right)
$$

Moreover, the relations (3.4) and (3.11) yield

$$
\dot{\rho}_{0}+(m-1) \rho_{0} \operatorname{tr} \mathbf{L}=0 \quad \text { and } \quad \dot{\mathbf{M}}+f \mathbf{P M}=\mathbf{0} .
$$


A gauge transformation is now introduced via

$$
\tilde{\mathbf{L}}=\mathbf{D L D}^{-1}+\frac{1}{2} f \mathbf{P}, \quad \tilde{\mathbf{E}}=\mathbf{D E D}^{-1},
$$

where

$$
\mathbf{D}=\exp \left(\frac{1}{2} \mathbf{P} f t\right)
$$

to obtain

$$
\dot{\tilde{\mathbf{E}}}+\tilde{\mathbf{E}} \tilde{\mathbf{L}}+\tilde{\mathbf{L}}^{T} \tilde{\mathbf{E}}+(m-1) \tilde{\mathbf{E}} \operatorname{tr} \tilde{\mathbf{L}}=\mathbf{0}
$$

and

$$
\dot{\tilde{\mathbf{L}}}+\tilde{\mathbf{L}}^{2}+\frac{1}{4} f^{2} \mathbf{I}+2 \varepsilon_{2}(t) \frac{m}{m-1} \tilde{\mathbf{E}}=\mathbf{0} .
$$

On use of the relation

$$
\mathbf{P H P}=\mathbf{H}^{T}-(\operatorname{tr} \mathbf{H}) \mathbf{I}
$$

together with the Cayley-Hamilton identity

$$
\tilde{\mathbf{L}}^{2}-(\operatorname{tr} \tilde{\mathbf{L}}) \tilde{\mathbf{L}}+(\operatorname{det} \tilde{\mathbf{L}}) \mathbf{I}=\mathbf{0}
$$

it is seen that (6.2) yields

$$
\dot{\tilde{\mathbf{L}}}+(\operatorname{tr} \tilde{\mathbf{L}}) \tilde{\mathbf{L}}-(\operatorname{det} \tilde{\mathbf{L}}) \mathbf{I}+\frac{1}{4} f^{2} \mathbf{I}+2 \varepsilon_{2}(t) \frac{m}{m-1} \tilde{\mathbf{E}}=\mathbf{0} .
$$

Moreover, on introduction of a new trace-free matrix $\tilde{\mathbf{Q}}$ according to

$$
\tilde{\mathbf{Q}}=\mathbf{P} \tilde{\mathbf{E}}
$$

the matrix equation (6.1) becomes

$$
\dot{\tilde{\mathbf{Q}}}+[\tilde{\mathbf{Q}}, \tilde{\mathbf{L}}]+m \tilde{\mathbf{Q}}(\operatorname{tr} \tilde{\mathbf{L}})=0 .
$$

Since $\operatorname{tr} \mathbf{L}=\operatorname{tr} \tilde{\mathbf{L}}=2 \dot{\Omega} / \Omega$, it is natural to introduce the scaling

$$
\overline{\mathbf{L}}=\tilde{\mathbf{L}} \Omega^{2}, \quad \overline{\mathbf{E}}=\tilde{\mathbf{E}} \Omega^{2 m}, \quad \overline{\mathbf{Q}}=\tilde{\mathbf{Q}} \Omega^{2 m},
$$

whence (6.3) and (6.4) reduce, in turn, to

$$
\dot{\overline{\mathbf{L}}}-\Omega^{-2}(\operatorname{det} \overline{\mathbf{L}}) \mathbf{I}+\frac{f^{2}}{4} \Omega^{2} \mathbf{I}+2 \varepsilon_{2}(t) \frac{m}{m-1} \Omega^{2(1-m)} \overline{\mathbf{E}}=\mathbf{0}
$$

and

$$
\dot{\overline{\mathbf{Q}}}+\Omega^{-2}[\overline{\mathbf{Q}}, \overline{\mathbf{L}}]=\mathbf{0},
$$

where

$$
\overline{\mathbf{L}}^{*}=\overline{\mathbf{L}}-\frac{1}{2}(\operatorname{tr} \overline{\mathbf{L}}) \mathbf{I}
$$


denotes the trace-free part of $\overline{\mathbf{L}}$. Moreover, the trace-free part of (6.5) yields

$$
\dot{\overline{\mathbf{L}}}^{*}+\varepsilon_{2}(t) \frac{m}{m-1} \Omega^{2(1-m)}[\overline{\mathbf{Q}}, \mathbf{P}]=\mathbf{0},
$$

while its trace gives

$$
(\operatorname{tr} \overline{\mathbf{L}})^{\cdot}-2 \Omega^{-2}\left(\operatorname{det} \overline{\mathbf{L}}^{*}\right)-\frac{1}{2} \Omega^{-2}(\operatorname{tr} \overline{\mathbf{L}})^{2}+\frac{1}{2} f^{2} \Omega^{2}+2 \varepsilon_{2}(t) \frac{m}{m-1} \Omega^{2(1-m)}(\operatorname{tr} \overline{\mathbf{E}})=0 .
$$

Insertion of the expression (3.20) for $\varepsilon_{2}$ (with $\alpha=1$ ) into (6.7) yields

$$
\dot{\overline{\mathbf{L}}}^{*}+\Omega^{-2}[\overline{\mathbf{Q}}, \mathbf{P}]=\mathbf{0}
$$

and on introduction of the new time measure $\tau$ according to

$$
d \tau=\Omega^{-2} d t
$$

the systems (6.6) and (6.9) become in turn

$$
\overline{\mathbf{Q}}^{\prime}+\left[\overline{\mathbf{Q}}, \overline{\mathbf{L}}^{*}\right]=\mathbf{0} \quad \text { and } \quad \overline{\mathbf{L}}^{* \prime}+[\overline{\mathbf{Q}}, \mathbf{P}]=\mathbf{0},
$$

where the prime denotes $d / d \tau$. The matrix system (6.10) constitutes the compatibility condition

$$
\mathcal{M}^{\prime}(\lambda)+[\mathcal{M}(\lambda), \mathcal{L}(\lambda)]=\mathbf{0}
$$

for the Lax pair

$$
\Psi^{\prime}=\mathcal{L}(\lambda) \Psi, \quad \mu \Psi=\mathcal{M}(\lambda) \Psi
$$

where

$$
\mathcal{L}(\lambda)=\overline{\mathbf{L}}^{*}+\lambda \mathbf{P}, \quad \mathcal{M}(\lambda)=\overline{\mathbf{Q}}+\lambda \overline{\mathbf{L}}^{*}+\lambda^{2} \mathbf{P} .
$$

An analogous result has been obtained in the case of non-conducting rotating gas clouds in [19]. As in that work, there is an interesting Steen-Ermakov connection. Thus, on setting

$$
\Sigma=\Omega^{-1}
$$

then the relation (6.8) is readily shown to reduce to a Steen-Ermakov equation, namely

$$
\Sigma^{\prime \prime}+\left(\operatorname{det} \overline{\mathbf{L}}^{*}-\operatorname{tr} \overline{\mathbf{E}}\right) \Sigma=\frac{f^{2}}{4 \Sigma^{3}} .
$$

Results of [19] related to the Lax pair for a spinning gas cloud system carry over mutatis mutandis to the Lax pair (6.11) obtained in the present magnetogasdynamic study. Thus, the linear system (6.11) is gauge equivalent to the standard Lax pair for the stationary reduction of the integrable cubic nonlinear Schrödinger equation. The connection may be made in the manner set down in [19].

\section{Conclusion}

It has been shown via an elliptic vortex ansatz that there is hidden integrable structure of Ermakov-Ray-Reid type underlying a 2+1-dimensional non-isothermal magnetogasdynamic system. The Ermakov variables turn out to have a natural physical interpretation as the semi-axes of the time-modulated density representation. Moreover, a Lax pair for the original nonlinear dynamical subsystem has been constructed. The preceeding and previous studies such as that in [4] suggest that a general investigation of the occurrence of integrable Ermakov-Ray-Reid structure in 2+1-dimensional hydrodynamic systems would be of interest. It is noted that Hamiltonian-Ermakov type systems have been additionally investigated in $[1,5]$. 


\section{References}

[1] Cerveró J.M., Lejarreta J.D., Ermakov Hamiltonians, Phys. Lett. A 156 (1991), 201-205.

[2] Dyson F.J., Dynamics of a spinning gas cloud, J. Math. Mech. 18 (1969), 91-101.

[3] Ermakov V.P., Second-order differential equations: conditions for complete integrability, Univ. Izv. Kiev 20 (1880), no. 9, 1-25.

[4] Ferapontov E.V., Khusnutdinova K.R., The characterization of two-component (2+1)-dimensional integrable systems of hydrodynamic type, J. Phys. A: Math. Gen. 37 (2004), 2949-2963, nlin.SI/0310021.

[5] Haas F., Goedert J., On the Hamiltonian structure of Ermakov systems, J. Phys. A: Math. Gen. 29 (1996), 4083-4092, math-ph/0211032.

[6] Neukirch T., Quasi-equilibria: a special class of time-dependent solutions of the two-dimensional magnetohydrodynamic equations, Phys. Plasmas 2 (1995), 4389-4399.

[7] Neukirch T., Cheung D.L.G., A class of accelerated solutions of the two-dimensional ideal magnetohydrodynamic equations, R. Soc. Lond. Proc. Ser. A Math. Phys. Eng. Sci. 457 (2001), 2547-2566.

[8] Neukirch T., Priest E.R., Generalization of a special class of time-dependent solutions of the two-dimensional magnetohydrodynamic equations to arbitrary pressure profiles, Phys. Plasmas 7 (2000), 3105-3107.

[9] Ovsiannikov L.V., A new solution of the hydrodynamic equations, Dokl. Akad. Nauk SSSR 111 (1956), $47-49$.

[10] Ray J.R., Nonlinear superposition law for generalized Ermakov systems, Phys. Lett. A 78 (1980), 4-6.

[11] Reid J.L., Ray J.R., Ermakov systems, nonlinear superposition, and solutions of nonlinear equations of motion, J. Math. Phys. 21 (1980), 1583-1587.

[12] Rogers C., A Ermakov-Ray-Reid reduction in 2+1-dimensional magnetogasdynamics, in Group Analysis of Differential Equations and Integrable Systems, Editors N.M. Ivanova, P.G.L. Leach, R.O. Popovych, C. Sophocleous, P.A. Damianou, Department of Mathematics and Statistics, University of Cyprus, Nicosia, 2011, 164-177.

[13] Rogers C., Elliptic warm-core theory: the pulsrodon, Phys. Lett. A 138 (1989), 267-273.

[14] Rogers C., An H., Ermakov-Ray-Reid systems in (2+1)-dimensional rotating shallow water theory, Stud. Appl. Math. 125 (2010), 275-299.

[15] Rogers C., Hoenselaers C., Ray J.R., On (2+1)-dimensional Ermakov systems, J. Phys. A: Math. Gen. 26 (1993), 2625-2633.

[16] Rogers C., Malomed B., An H., Ermakov-Ray-Reid reductions of variational approximations in nonlinear optics, Stud. Appl. Math., to appear.

[17] Rogers C., Malomed B., Chow K., An H., Ermakov-Ray-Reid systems in nonlinear optics, J. Phys. A: Math. Theor. 43 (2010), 455214, 15 pages.

[18] Rogers C., Schief W.K., Multi-component Ermakov systems: structure and linearization, J. Math. Anal. Appl. 198 (1996), 194-220.

[19] Rogers C., Schief W.K., On the integrability of a Hamiltonian reduction of a 2+1-dimensional non-isothermal rotating gas cloud system, Nonlinearity 24 (2011), 3165-3178.

[20] Rogers C., Schief W.K., The pulsrodon in 2+1-dimensional magneto-gasdynamics: Hamiltonian structure and integrability, J. Math. Phys. 52 (2011), 083701, 20 pages.

[21] Schäfer G., Gravity-wave astrophysics, in Relativistic Gravity Research with Emphasis on Experiments and Observations, Lecture Notes in Physics, Vol. 410, Springer-Verlag, Berlin, 1992, 163-183.

[22] Steen A., Om Formen for Integralet af den lineare Differentialligning af anden Orden, Forhandl. af Kjobnhaven (1874), 1-12. 